\title{
Tests of Perturbative QCD with Photon Final States at the ATLAS Experiment
}

\author{
B. Vachon, on behalf of the ATLAS Collaboration* \\ McGill University \\ E-mail: Brigitte.Vachon@McGill.ca \\ on behalf of the ATLAS Collaboration
}

\begin{abstract}
The production of prompt isolated photons at hadron colliders provides a stringent test of perturbative Quantum Chromodynamics and can be used to probe the gluon density function of the proton. Using proton-proton collision data provided by the Large Hadron Collider, the ATLAS collaboration has performed numerous cross-section measurements of prompt photon production, among which are a precise measurement of the production of isolated prompt photons in association with heavy flavor jets, a first measurement of the production cross-section of tri-photon final states at a center of mass energy of $8 \mathrm{TeV}$, and a photon plus jet cross-section measurement at $13 \mathrm{TeV}$. The results are compared with state-of-the-art theory predictions, indicating several interesting discrepancies.
\end{abstract}

The 39th International Conference on High Energy Physics (ICHEP2018)

4-11 July, 2018

Seoul, Korea

* Speaker. 


\section{Introduction}

Studies of physics processes resulting in the production of at least one photon in the final state are important for several reasons. These studies provide the ability to test Standard Model predictions in new kinematic regimes using a unique colourless probe, to further constrain the constituents of the proton, and to provide a description of the kinematics of background events for different searches for new physics. The most recent results of the study of processes with a photon in the final state obtained using the ATLAS detector [1] at the Large Hadron Collider (LHC) [2] are presented here.

In all the measurements presented below, "prompt" signal photons are defined as those originating from the hard scatter and not from hadron decays. Photons candidates are reconstructed from clusters of energy deposited in the ATLAS electromagnetic calorimeter and are classified as unconverted or converted photons. Additional requirements on the shape of the electromagnetic shower are applied in order to reject hadronic jet background. Identified photons are also required to be isolated to further suppress background coming from jets mis-identified as a photon.

\section{Results}

In proton-proton collisions, isolated prompt photons are predominantly produced through a Quantum Chromodynamics (QCD) Compton scattering process either directly or via fragmentation processes. As a result, these measurements are sensitive, in particular, to the gluon content of the proton.

Inclusive prompt photon production differential cross-sections at a centre-of-mass of $13 \mathrm{TeV}$ have been recently measured [3]. The measurements are performed in different regions of photon pseudorapidity, and reach up to photon transverse energy of approximately $1.5 \mathrm{TeV}$. Next-toleading (NLO) order predictions provide overall a reasonable description of the measurements, however, theoretical uncertainties are significantly larger than the experimental uncertainties, limiting the ability to test perturbative QCD and further constrain the proton parton distribution functions (PDF). Next-to-next-to-leading-order calculations have recently been made available [4,5] and the theoretical uncertainties on these calculations are now of the same order as the experimental uncertainties. This opens up the possibility to include prompt photon data into PDF fits, which has been shown [5] to lead to a moderate reduction of the gluon PDF uncertainties at medium- $x$ and a preference for a somewhat smaller central value at large- $x$.

The total fiducial and differential cross-sections for di-photon production have been measured at a proton-proton centre-of-mass energy of $8 \mathrm{TeV}$ [6]. Due to improvements in the background estimation, systematic uncertainties are reduced by a factor of 2 compared to those obtained at 7 $\mathrm{TeV}$ [7]. The best description of the total fiducial cross-section measurement is obtained using matrix element calculations at NLO combined with parton shower. Fixed-order calculations at next-to-next-to-leading order (NNLO) underestimate the total fiducial cross-section. Differential cross-sections have been measured as function of six observables, and are well-described by computations combining NLO matrix elements matched to parton shower. Some specific regions of phase space are particularly sensitive to soft-gluon emissions, such as the low $a_{T}$ region, where the variable $a_{T}$ is defined as the transverse component of the $p_{T}$ of the di-photon system with respect 
to the thrust axis. A good understanding of this low $a_{T}$ region constitutes an important step for perturbative QCD resummation techniques aiming to describe more complicated processes, e.g. those involving colored final states. In this region, the inclusion of soft-gluon resummation or the use of parton shower allow to obtain a correct description of data.

Measurements of the total fiducial and differential cross-sections of the production of at least three photons in the final state have been performed at $8 \mathrm{TeV}$ [8]. These measurements cover a broad range of kinematic regions which are complementary to those explored by the study of inclusive single and di-photon production processes. Next-to-leading-order QCD predictions underestimate the measured total fiducial cross-section by approximately a factor of 2 . The addition of a parton shower to the fixed-order calculations is shown to improve slightly the agreement with the measured value. Differential cross-sections are measured as function of 13 kinematic variables. These measurements are compared with state-of-the art NLO calculations with and without a parton shower. The measurements indicate the need for improved modelling of this process.

The dynamic of photon+jets final states has been studied at a centre-of-mass energy of $13 \mathrm{TeV}$ [9]. Differential cross-sections are measured as function of five kinematic variables and compared to fixed-order calculations at NLO accuracy. Overall, these NLO calculations provide a good description of the measurements, although theoretical uncertainties are larger than experimental uncertainties. The photon+jets final states are primarily produced through the $q g \rightarrow q \gamma$ process that proceeds through the exchange of a quark. Data are shown to be consistent with the dominance of processes in which the exchanged particle is a quark.

First measurements of the production of photon $+b$ and photon $+c$ quark in proton-proton collisions have recently been published [10]. The production of isolated-photon plus heavy-flavour is sensitive to the $b$ and $c$ quark content of the proton, through the Compton process. The gluon spitting contribution to these final states increases at higher photon energy, and it dominates the photon $+b$ production. The measured photon $+b$ cross-sections indicate that the NLO predictions obtained using a four-flavour scheme underestimate the data at high $E_{\mathrm{T}}^{\gamma}$. This is consistent with the expectation that the four-flavour scheme is better suited for energies close to the $b$-quark mass. Predictions obtained using the five-flavour scheme describe the data better at higher $E_{\mathrm{T}}^{\gamma}$, but also underestimate data by up to a factor of 2 at the very highest $E_{\mathrm{T}}^{\gamma}$ measured. Since the high $E_{\mathrm{T}}^{\gamma}$ region is where the gluon-splitting contribution is expected to become more significant relative to the Compton contribution, this indicates that higher-order calculations would seemingly be needed for a better description of the data in that high $E_{\mathrm{T}}^{\gamma}$ region. Within the total measurements uncertainty, the measured photon $+c$ differential cross-sections are consistent with leading-order and next-toleading order predictions. Theoretical predictions obtained using PDF sets that include small intrinsic charm [11,12] contributions predict higher cross-section values in the forward region at $E_{\mathrm{T}}^{\gamma}$ $>105 \mathrm{GeV}$, than those using the nominal PDF sets (by up to a factor of 1.5). A reduction in measurement uncertainty in this region would make it possible to constrain the existence of intrinsic charm in the proton.

\section{Summary}

In summary, cross-section measurements of known Standard Model processes with photon final states obtained using the ATLAS detector at the LHC have been discussed. These measure- 
ments provide stringent tests of QCD and indicate the need for calculations beyond NLO in order to reduce theoretical uncertainties and improve the modelling of these processes. These measurements can eventually be used to further constrain the make-up of the proton.

\section{Acknowledgments}

The author wishes to thank members of the organizing and program committees of the 39th International Conference on High Energy Physics (ICHEP2018) for the preparation and hosting of a stimulating and fruitful meeting.

\section{References}

[1] ATLAS Collaboration, The ATLAS Experiment at the CERN Large Hadron Collider, JINST 3 (2008) S08003.

[2] L. Evans and P. Bryant (editors), LHC Machine, JINST 3 (2008) S08001.

[3] ATLAS Collaboration, Measurement of the cross section for inclusive isolated-photon production in pp collisions at $\sqrt{s}=13 \mathrm{TeV}$ using the ATLAS detector, Phys. Lett. B 770 (2017) 473 [hep-ex/1701.06882].

[4] J. M. Campbell, R. K. Ellis and C. Williams, Direct photon production at next-to-next-to-leading order, Phys. Rev. Lett. 118 (2017) 222001 [hep-ph/1612.04333].

[5] J. M. Campbell, J. Rojo, E. Slade and C. Williams, Direct photon production and PDF fits reloaded, Eur. Phys. J. C 786 (2018) 470 [hep-ph/1802 . 03021$].$

[6] ATLAS Collaboration, Measurements of integrated and differential cross sections for isolated photon pair production in pp collisions at $\sqrt{s}=8 \mathrm{TeV}$ with the ATLAS detector, Phys. Rev. D 95 (2017) 112005 [hep-ex/1704.03839].

[7] ATLAS Collaboration, Measurement of isolated-photon pair production in pp collisions at $\sqrt{s}=$ 7 TeV with the ATLAS detector, JHEP 01 (2013) 086 [hep-ex/1211. 1913].

[8] ATLAS Collaboration, Measurement of the productionâLZŽcross section of three isolated photons in pp collisions at $s=8 \mathrm{TeV}$ using the ATLAS detector, Phys. Lett. B 781 (2018) 55 [hep-ex/1712.07291].

[9] ATLAS Collaboration, Measurement of the cross section for isolated-photon plus jet production in pp collisions at $\sqrt{s}=13 \mathrm{TeV}$ using the ATLAS detector, Phys. Lett. B 780 (2018) 578 [hep-ex/1801.00112].

[10] ATLAS Collaboration, Measurement of differential cross sections of isolated-photon plus heavy-flavour jet production in pp collisions at $\sqrt{s}=8 \mathrm{TeV}$ using the ATLAS detector, Phys. Lett. B 776 (2018) 295 [hep-ex/1710.09560].

[11] S. Brodsky, P. Hoyer, C. Peterson and N. Sakai, The intrinsic charm of the proton, Phys. Lett. B 93 (1980) 451.

[12] S. Brodsky, C. Peterson, N. Sakai, Intrinsic heavy-quark states, Phys. Rev. D 23 (1981) 2745. 